\title{
Time-Based Extensions to Adaptation Techniques $^{\star}$
}

\author{
Mária Bieliková and Rastislav Habala \\ Institute of Informatics and Software Engineering \\ Faculty of Informatics and Information Technologies \\ Slovak University of Technology \\ Ilkovičova 3, 84216 Bratislava, Slovakia \\ http://www.fiit.stuba.sk/ bielik
}

\begin{abstract}
In this paper we present an approach to time-based adaptation in adaptive hypermedia systems. Time is used as a part of the context (or environment) model. We have proposed extensions of known adaptation techniques by means of the notion of time. We experimented with proposed extensions and implemented a software system called TIM, which adapts presentation of educational module administrative information according user characteristics and a context represented by time of information presentation.
\end{abstract}

\section{Introduction}

An important feature of effective delivery of information is adaptivity based on the presentation context. The presentation context is defined as a collection of data which depend on the current state of the presentation. It is often described using technology or user platform adaptation dimension (hardware, software, network bandwidth) or external environment adaptation dimension (user location, language, socio-political issues) [3]. We emphasize one of important characteristics to the context - time. Time is fundamental characteristic since the context and user characteristics are unstable and changing.

Our approach is based on the fact that some information is not valid forever. Presented information has defined an initial time of validity and/or end time of validity [2]. Moreover, during the validity time, relevance of this information can evolve. The task of the adaptive hypermedia $(\mathrm{AH})$ system is to observe current time of information presentation besides other characteristics (user knowledge, preferences, etc.). Time constrained adaptation based on a time of the day is used in tourist guide adaptive systems $([4,7])$. The opening times of attractions represent important characteristic used in adaptation of the city guide.

We also support the domain model changes. Often the domain model evolves in time (e.g., new information is added or existing information is corrected). It is important to be able to observe this evolution. The $\mathrm{AH}$ system in this case

\footnotetext{
* This work has been partially supported by the Grant Agency of Slovak Republic grant No. VG1/ 0162/03.
} 
serves a user the presentation of evolving information (e.g., changes of the content which occurred from the last user access to the AH system are highlighted).

Other options to exploiting time in adaptive hypermedia include targeting limited capacity of the human memory [1] or to provide models of versioning related to time (also known in software engineering as revisions [5]).

In this paper we concern time-based adaptive presentation and drawing user attention to domain model changes. We describe the most interesting extensions to known adaptation techniques with considering the time dimension. Proposed extensions are based on the model of time, which maps a real time to a symbolic time and provides necessary operations for working with both of them. Two basic structures form the basis of the time model: intervals and scopes.

Model of time is advantageous especially in cases when the domain model is going to be reused in various time periods, i.e. similar content is presented within various time intervals. Typical example of such functionality is a webbased whiteboard of university courses, presentation of scientific conferences or other professional repetitive activities. The domain model reuse in next execution of the event (be it university course or scientific conference) requires only settingup the intervals' initial and end times of validity in the model of time.

\section{Time-Based Adaptive Presentation}

Inserting/removing fragments. Presentation of a fragment is determined according time (along other characteristics represented in user or context models). Obvious technique is to define meta-data related to the fragment, which serve for adaptation. Adaptation rules define adaptive fragment inserting or removing in dependence of time.

Simplest implementation uses attributes from and to, which represent simple time dependent rule: the fragment is presented only if current time is in the interval defined by from and to values. If the value of these attributes (real time or symbolic time) is not defined, we consider infinity instead.

Sorting fragments. Sort criteria are extended by time (real or symbolic). This technique is especially useful for application domain of adaptive electronic whiteboards. Typical fragments that should be sorted by time are organized as a list of announcements or messages to the users.

Sorting fragments technique is used in our AH system TIM along with the collects relation. The collects relation enables effective wrapping of evolving list of fragments. Adding a new element into the list does not require any modification of the concept representing the list.

Change emphasizing. For effective comprehension of evolving information it is important to have a mechanism for adaptive presentation according changes, which occurred from the last user visit and to which the user should pay attention. We proposed the technique as follows. The adaptation engine compares the content of presented fragment that has been valid in time of the last presentation of the fragment and its current content. The distinctions are determined and emphasized to the user. Moreover, changes can be propagated to the parents of the 
changed fragment (i.e., the change is presented also in fragments, which occur on a path from the starting fragment to the changed fragment). The changes are indicated by annotating links leading to the changed fragment.

Meaningful change discovery is not an easy task. The problem is automatic recognition of the change significance. Often the change presents spell correction. Some other time the content is renewed but it is not necessary to read it again. We implemented this technique with explicit tagging in the changed content. The changed region is enclosed to the block with assigned time of change. For enabling described feature we proposed technique for time versioning of models (used here for domain model). This technique enables us to determine the state and the content of each concept in arbitrary time in the history. It is a base for accomplishing a comparison of the content of two snapshots of the concept.

Change emphasizing was recognized as extremely useful feature of our $\mathrm{AH}$ system for students who periodically visited the course information during term. For visual change highlighting we use a change of the background color. It is possible to use this technique in combination with known adaptation techniques. For example, links inside the changed content can be annotated (colored). We use a change of the background color for emphasizing unlike the approach of Hothi et al. [6] where the background color change serves for dimming fragments.

\section{Adaptive Navigation}

Adaptive link sorting and hiding. Both techniques are extended and implemented alike sorting and inserting/removing fragments. Links are sorted also considering the time criterion. For example, fresh concepts are presented first. Sorting can be based on concept creation time or concept validity time. Adaptive link hiding regards also time while decision about the link presentation is performed (along other characteristics represented in user or context models).

Adaptive link annotation. Time-based adaptive link annotation supports increasing of attention to a change, which occurred in the concept representing a target of the link. We distinguish two types of changes. Providing the concept content has changed or a new fragment has been added to the concept content it is denoted as "change". Other case is adding a new fragment using the collects relation. This fact is visualized by annotating by "new" icon (it reflects semantics of the collects relation and its intended use for e-whiteboard systems).

Adaptive link annotation by denoting changes or news is propagated. We have proposed also a mechanism for controlling propagation. Sometimes it is necessary to stop the change propagation because as a result we will see the change indication in many places, which can confuse the user.

\section{Conclusions}

In this paper we presented time constrained adaptation techniques. Our approach enables adaptive presentation of time dependent information, tracking evolution of application domain model in time together with support of drawing 
the user's attention to the content changes and time-based content versioning. Important characteristic of proposed approach is the support of a domain model reuse in various time periods.

We developed a time-based AH web-based system called TIM (TIMe-Aware Adaptive Hypermedia System). TIM has been first time applied in summer term 2002/03 to the Principles of Software Engineering (PSE) course with about 130 students enrolled. TIM serves the students in two ways: it features as a leading familiarization with a course and it serves as a presentation of the course running (and evolving) information.

We evaluated the TIM from several points of view. First, the system demonstrated as an appropriate tool for presenting evolving information. Invaluable feature for the content developer is the domain model reuse (in subsequent years of the course offering). As the most frequent problem indicated by students was known problem with adaptive web-based systems - the back button. The user expects specific behavior when pressing "back" in a browser menu. However, in adaptive systems (in time-aware system especially) there is no conventional back because the state of presentation already changed. Even though the system has provides own back and forward buttons with expected semantics, the students used these buttons infrequently.

The most valuable feature designated by students was change propagation. The emphasizing based on highlighting background was proved effective similarly to dimming adaptation technique based on shading background [6].

Our future work concerns the research of time-based adaptation in different application domains together with improving mechanisms for debugging defined models by simulation of time pass.

\section{References}

1. Peter Ágh and Mária Bieliková. Improving learning using cognitive characteristics of human memory, 2002. Tech. report. Slovak University of Technology in Bratislava.

2. Mária Bieliková. Adaptive presentation of evolving information using XML. In T. Okamoto et al., editors, Proc. of IEEE Int. Conf. of Advanced Learning Technologies - ICALT'2001, pages 193-196, Madison, USA, 2001. IEEE Press.

3. Mario Cannataro, Alfredo Cuzzocrea, and Andrea Pugliese. XAHM: an adaptive hypermedia model based on XML. In Proc. SEKE'02, pages 627-634, Ischia, Italy, July 2002. ACM Press.

4. Keith Cheverst, Keith Mitchell, and Nige Daies. The role of adaptive hypermedia in a context-aware tourist GUIDE. Communications of the ACM, 45(5):47-51, 2002.

5. Reidar Conradi and Bernhard Westfechtel. Version models for software configuration management. ACM Computing Surveys, 30(2):232-282, June 1998.

6. Jatinder Hothi, Wendy Hall, and Tim Sly. A study comparing the use of shaded text and adaptive navigational support in adaptive hypermedia. In C. Strapparava P. Brusilovsky, O. Stock, editors, Proc. of Int. Conf. on Adaptive Hypermedia and Adaptive Web-Based Systems, pages 335-342, Italy, 2000. Springer LNCS 1892.

7. Daniela Petrelli et al. Modelling and adapting to context. Personal and Ubiquitous Computing, 5:20-24, 2001. 